\title{
Ghost free dual vector theories in $2+1$ dimensions
}

\author{
D. Dalmazi \\ UNESP - Campus de Guaratinguetá - DFQ \\ Av. Dr. Ariberto Pereira da Cunha, 333 \\ CEP 12516-410 - Guaratinguetá - SP - Brazil. \\ E-mail: dalmazi@feg.unesp.br
}

November 5, 2018

\begin{abstract}
We explore here the issue of duality versus spectrum equivalence in abelian vector theories in $2+1$ dimensions. Specifically we examine a generalized self-dual (GSD) model where a Maxwell term is added to the self-dual model. A gauge embedding procedure applied to the GSD model leads to a Maxwell-Chern-Simons (MCS) theory with higher derivatives. We show that the latter contains a ghost mode contrary to the original GSD model. On the other hand, the same embedding procedure can be applied to $N_{f}$ fermions minimally coupled to the self-dual model. The dual theory corresponds to $N_{f}$ fermions with an extra Thirring term coupled to the gauge field via a Pauli-like term. By integrating over the fermions at $N_{f} \rightarrow$ $\infty$ in both matter coupled theories we obtain effective quadratic theories for the corresponding vector fields. On one hand, we have a nonlocal type of the GSD model. On the other hand, we have a nonlocal form of the MCS theory. It turns out that both theories have the same spectrum and are ghost free. By figuring out why we do not have ghosts in this case we are able to suggest a new master action which takes us from the local GSD to a nonlocal MCS model with the same spectrum of the original GSD model and ghost free. Furthermore, there is a dual map between both theories at classical level which survives quantum correlation functions up to contact terms. The remarks made here may be relevant for other applications of the master action approach.
\end{abstract}

PACS-No.: 11.15.-q, 11.10.Kk, 11.10.Gh, 11.10.Ef 


\section{Introduction}

The bosonization program in two dimensions is a good example of the power of dual descriptions of the same theory [1, 2], see also [3] and references therein. Although much less progress has been done in this area in higher dimensions, some nonperturbative features like confinement can also be revealed with the help of duality as in [4]. Here we are interested in the intermediate case of $2+1$ dimensions. By means of a master action it was shown in [5] that the gauge invariant sector of the Maxwell Chern-Simons (MCS) theory is on shell equivalent to the self-dual (SD) theory of [6] with the dual map $f_{\mu} \leftrightarrow \frac{\epsilon_{\mu \nu \alpha} \partial^{\nu} A^{\alpha}}{m}$. This type of duality is intimately related to the bosonization program in $d=2+1$ [7, 8, 9, 10, 11, 12].

The master action of [5] can be generalized to include matter fields as in [13] and [14, 15. Although very useful, specially at quantum level, the master action is not derived from first principles and it is justified a posteriori. A systematic derivation of dual gauge theories would certainly be welcome. In [14] a Noether gauge embedding procedure was suggested and applied in a variety of examples and dimensions [16, 17, 18]. However, it has been pointed out in [19] that this procedure may lead to ghosts in the dual gauge theory which has been explicitly verified in a dual model to a four dimensional Lorentz violating electrodynamics [20]. An understanding of this issue from the point of view of master actions allows us to propose an alternative master action to avoid the problem which is the aim of this work. We start in the next section from a rather general quadratic theory for a vector field and calculate the residue of its propagator around its poles in order to verify the presence of ghost modes. The so called Noether embedding procedure is briefly reviewed as well as its use to generate a dual gauge theory. In section 3 we compare the spectrum of a GSD theory of non-local type and its dual gauge model. Both theories are generated by a $N_{f} \rightarrow \infty$ limit of parity invariant fermions $(4 \times 4$ representation) coupled to vector fields. The equivalence of both spectra in this case leads us to formulate a new master action in section 4 to solve the problem of ghosts in the dual theory. In the last section we draw some conclusions.

\section{The GSD model and its higher derivative dual}

Allowing arbitrary functions of the D'Alambertian $a_{i}=a_{i}(\square)$, the Lagrangian below includes a rather general set of Poincaré covariant quadratic theories for an abelian vector field in $d=2+1$, see also [21, 18, 


$$
\mathcal{L}_{G S D}=a_{0} f^{\mu} f_{\mu}+a_{1} \epsilon_{\alpha \beta \gamma} f^{\alpha} \partial^{\beta} f^{\gamma}+\frac{a_{2}}{2} F_{\mu \nu}(f) F^{\mu \nu}(f)
$$

We use $g_{\mu \nu}=(+,-,-)$ and assume in this work that $a_{i}$ are constants but later on we will make a comment on the general case. Minimizing $\mathcal{L}_{G S D}$ we can write its equations of motion in a self-dual form where $\tilde{f}$ defined below stands for the dual field:

$$
f_{\mu}=\frac{a_{1}}{a_{0}} E_{\mu \nu} f^{\nu}+\frac{a_{2}}{a_{0}} \square \theta_{\mu \nu} f^{\nu} \equiv \tilde{f}_{\mu}
$$

where $E_{\mu \nu}=\epsilon_{\mu \nu \gamma} \partial^{\gamma}$ and $\square \theta_{\mu \nu}=g_{\mu \nu}-\partial_{\mu} \partial_{\nu}$. From (2) we get $\partial_{\mu} f^{\mu}=0$. Since $E_{\mu \nu} E^{\nu \gamma}=$ $-\square \theta_{\mu}^{\gamma}$ and both $a_{0}$ and $a_{1}$ are assumed to be non-vanishing, it is easy to derive from (2):

$$
\left[\left(a_{0}-a_{2} \square\right)^{2}+a_{1}^{2} \square\right] f^{\mu}=a_{2}^{2}\left(\square+m_{+}^{2}\right)\left(\square+m_{-}^{2}\right) f^{\mu}=0
$$

with

$$
2 m_{ \pm}^{2}=b^{2}-2 a \pm \sqrt{\left(b^{2}-2 a\right)^{2}-4 a^{2}}
$$

and $a=a_{0} / a_{2}, b=a_{1} / a_{2}$. The masses are real for $a<0$ or $b^{2}>4 a$ if $a>0$. An interesting observation is that we can not have $\left(a_{0}-a_{2} \square\right) f^{\mu}=0$, since from (3) we see that this would lead to $\square f^{\mu}=0$ and this two equations together would contradict our hypothesis $a_{0} \neq 0$. We can read off the propagator for the generalized self-dual field from (11). In the momentum space we have:

$$
\left\langle f_{\alpha}(k) f_{\beta}(-k)\right\rangle_{G S D}=\frac{g_{\alpha \beta}-\theta_{\alpha \beta}}{a_{0}}+\frac{\left(a_{0}+k^{2} a_{2}\right)}{\left(a_{0}+k^{2} a_{2}\right)^{2}-a_{1}^{2} k^{2}} \theta_{\alpha \beta}+\frac{i a_{1} E_{\alpha \beta}}{\left(a_{0}+k^{2} a_{2}\right)^{2}-a_{1}^{2} k^{2}}
$$

As expected from (3) there are two simple poles at $k^{2}=m_{ \pm}^{2}$ which become a double pole only at the special point $a_{1}^{2}=4 a_{0} a_{2}$. In order to make sure that we are free of ghosts we have to show in general, see e.g. [20, 21, 22], that the imaginary part of the residue of the propagator at each pole is positive when saturated with conserved currents, that is $\operatorname{Im}\left(\operatorname{Res}\left(J_{\alpha}\left\langle f^{\alpha}(k) f^{\beta}(-k)\right\rangle J_{\beta}^{*}\right)\right)>0$ with $k_{\mu} J^{\mu}=0=k^{\mu} J_{\mu}^{*}$. In $d=2+1$ the propagator of a vector field in a Poincarè covariant theory will have the general Lorentz structure $A\left(k^{2}\right)\left(g_{\mu \nu}-\theta_{\mu \nu}\right)+B\left(k^{2}\right) \theta_{\mu \nu}+C\left(k^{2}\right) E_{\mu \nu}$. In practice ${ }^{1}$, this is equivalent to

\footnotetext{
${ }^{1}$ By choosing a convenient frame for a massive pole $k=(k, 0,0)$, which implies $J_{\mu}=$ $\left(0, J_{1}, J_{2}\right)$, and using the general formula for a propagator in $d=2+1$ one can show that $\operatorname{Im}\left(\operatorname{Res}\left(J_{\alpha}\left\langle f^{\alpha}(k) f^{\beta}(-k)\right\rangle J_{\beta}^{*}\right)\right)=-S J \bar{J}=-S\left(J_{1}+i J_{2}\right)\left(J_{1}^{*}-i J_{2}^{*}\right)$ where for every massive single pole $S=\lim _{k^{2} \rightarrow m^{2}}\left(k^{2}-m^{2}\right) B\left(k^{2}\right)$.
} 
require $\lim _{k^{2} \rightarrow m^{2}}\left(k^{2}-m^{2}\right) B\left(k^{2}\right)<0$. Applying this requirement at $k^{2}=m_{+}^{2}$ and $k^{2}=m_{-}^{2}$ respectively we have:

$$
\begin{aligned}
& \frac{a+m_{+}^{2}}{a_{2}\left(m_{+}^{2}-m_{-}^{2}\right)}=\frac{b^{2}+\sqrt{b^{2}\left(b^{2}-4 a\right)}}{2 a_{2}\left(m_{+}^{2}-m_{-}^{2}\right)}<0 \\
& \frac{a+m_{-}^{2}}{a_{2}\left(m_{-}^{2}-m_{+}^{2}\right)}=-\frac{b^{2}-\sqrt{b^{2}\left(b^{2}-4 a\right)}}{2 a_{2}\left(m_{+}^{2}-m_{-}^{2}\right)}<0
\end{aligned}
$$

Since $m_{+}^{2}>m_{-}^{2}$ we are free of ghosts if $a_{0}>0$ and $a_{2}<0$, in agreement with [21]. Those are in fact usual conditions on the mass term of the self-dual model and on the coefficient of the Maxwell term in QED in $d=2+1$ respectively. Notice that we get rid automatically of the double pole under such circumstances.

In [18] the gauge theory dual to $\mathcal{L}_{G S D}$ was derived via a Noether gauge embedding procedure which in summary works as follows. Since the equations of motion for the the self-dual field come from $K^{\mu}=2\left(a_{0} f^{\mu}-a_{1} E^{\mu \nu} f_{\nu}-a_{2} \square \theta^{\mu \nu} f_{\nu}\right)=0$, and under a local $U(1)$ transformation $\delta^{\phi} f_{\mu}=\partial_{\mu} \phi$ we have $\delta^{\phi} K_{\mu}=2 a_{0} \delta^{\phi} f_{\mu}$. Thus, if we define $\mathcal{L}_{H D-M C S}=\mathcal{L}_{G S D}-K_{\alpha} K^{\alpha} / 4 a_{0}$ it follows that $\delta^{\phi} \mathcal{L}_{H D-M C S}=0$ and we have a gauge invariant theory. By minimizing this theory $\delta \mathcal{L}_{H D-M C S}=K^{\mu} \delta f_{\mu}-K^{\mu} \delta K_{\mu} / 2 r_{0}=0$ it is clear that the equations of motion of $\mathcal{L}_{G S D}\left(K_{\mu}=0\right)$ lead to $\delta \mathcal{L}_{H D-M C S}=0$ and therefore are embedded in the equations of motion of the new gauge theory. We stress that such embedding does not guarantee equivalence of the equations of motion of both theories, not even in the gauge invariant sector of $\mathcal{L}_{H D-M C S}$. Applying this embedding explicitly, renaming the field $f_{\mu} \rightarrow A_{\mu}$, one has the higher derivative theory [18] :

$$
\begin{aligned}
\mathcal{L}_{H D-M C S} & =\mathcal{L}_{G S D}-\frac{K_{\alpha} K^{\alpha}}{4 a_{0}} \\
& =A^{\mu} a_{1}\left(1-\frac{2 a_{2} \square}{a_{0}}\right) \epsilon_{\mu \nu \gamma} \partial^{\gamma} A^{\nu}-\frac{1}{2} F_{\mu \nu}(A)\left(\frac{a_{1}^{2}}{a_{0}}-\frac{a_{2}^{2} \square}{a_{0}}+a_{2}\right) F^{\mu \nu}(A)(8)
\end{aligned}
$$

The equations of motion of the higher derivative model (8) can be written as

$$
a_{1}\left(1-\frac{2 a_{2} \square}{a_{0}}\right) \epsilon_{\mu \nu \gamma} \partial^{\gamma} A^{\nu}+\square\left(\frac{a_{1}^{2}}{a_{0}}-\frac{a_{2}^{2} \square}{a_{0}}+a_{2}\right) \theta_{\mu \nu} A^{\nu}=a_{0}\left(\tilde{A}_{\mu}-\tilde{\tilde{A}}_{\mu}\right)=0,
$$

which makes evident, see (2), the duality at classical level with the GSD model upon replacing $f_{\mu}$ by $\tilde{A}_{\mu}$. From (9) we deduce the analogous of formula (3):

$$
\left(a_{1}^{2}+a_{2}^{2} \square\right)\left(\square+m_{+}^{2}\right)\left(\square+m_{-}^{2}\right) \epsilon_{\mu \nu \gamma} \partial^{\gamma} A^{\nu}=0
$$


The expression (10) shows that we have new classical solutions in the gauge invariant sector $\left(a_{1}^{2}+a_{2}^{2} \square\right) \epsilon_{\mu \nu \gamma} \partial^{\gamma} A^{\nu}=0$ which were not present in the GSD model. The new solutions will correspond to a new pole in the propagator. After introducing a gauge fixing term we have the propagator:

$$
\left\langle A_{\alpha}(k) A_{\beta}(-k)\right\rangle_{H D-M C S}=\frac{g_{\alpha \beta}-\theta_{\alpha \beta}}{\lambda k^{2}}+B\left(k^{2}\right) \theta_{\alpha \beta}+\frac{i a_{0} a_{1}\left(a_{0}+2 a_{2} k^{2}\right) E_{\alpha \beta}}{k^{2}\left(a_{1}^{2}-k^{2} a_{2}^{2}\right)\left[\left(a_{0}+k^{2} a_{2}\right)^{2}-a_{1}^{2} k^{2}\right]}
$$

where, in agreement with the general predictions of [19], we have

$$
B\left(k^{2}\right)=\frac{\left(a_{0}+k^{2} a_{2}\right)}{\left(a_{0}+k^{2} a_{2}\right)^{2}-a_{1}^{2} k^{2}}-\frac{a_{2}}{a_{2}^{2} k^{2}-a_{1}^{2}}
$$

Since the first term in $B\left(k^{2}\right)$ is the same one appearing in (5) it is clear that $k^{2}=m_{ \pm}^{2}$ are still physical poles as in the GSD model for $a_{0}>0$ and $a_{2}<0$. However, since $\lim _{k^{2} \rightarrow\left(a_{1} / a_{2}\right)^{2}}\left[k^{2}-\left(a_{1} / a_{2}\right)^{2}\right] B\left(k^{2}\right)=-1 / a_{2}$ should be negative the new pole will be a ghost if we insist in $a_{2}<0$. On the other hand, if we drop that condition one of the poles $k^{2}=m_{ \pm}^{2}$ will become a ghost mode. From (12) we see that the only possible exit might be a fine tuning of $a_{0}, a_{1}, a_{2}$ such that one of the poles $m_{ \pm}^{2}$ coincides with $\left(a_{1} / a_{2}\right)^{2}$ and the corresponding residue has the right sign. This is only possible for $a_{1}^{2}=-\left(a_{0} a_{2}\right) / 2$. In this case $m_{-}^{2}=\left(a_{1} / a_{2}\right)^{2}$ but $B\left(k^{2}\right)=-a_{0} /\left[a_{2}^{2}\left(k^{2}-m_{-}^{2}\right)\left(k^{2}-m_{+}^{2}\right)\right]$ which implies that one of the poles $m_{ \pm}^{2}$ must be a ghost again. In conclusion, the dual gauge model $\mathcal{L}_{H D-M C S}$ obtained by the Noether embedding procedure will always contain a ghost in the spectrum as far as $a_{2} \neq 0$. We only recover a ghost free theory in the well known case of the SD/MCS duality [5] where $a_{2}=0$. For future use we notice that in [18] a master action which relates $\mathcal{L}_{G S D}$ and $\mathcal{L}_{H D-M C S}$ was suggested:

$$
\begin{aligned}
\mathcal{L}_{\text {Master }} & =a_{0} f^{\mu} f_{\mu}+a_{1} \epsilon_{\alpha \beta \gamma} f^{\alpha} \partial^{\beta} f^{\gamma}+\frac{a_{2}}{2} F_{\mu \nu}(f) F^{\mu \nu}(f) \\
& -a_{1} \epsilon_{\alpha \beta \gamma}\left(A^{\alpha}-f^{\alpha}\right) \partial^{\beta}\left(A^{\gamma}-f^{\gamma}\right)-\frac{a_{2}}{2} F_{\mu \nu}(A-f) F^{\mu \nu}(A-f)
\end{aligned}
$$

The existence of a interpolating master action makes even more surprising the equivalence of two partition functions of theories with different spectrum. In the next section $N_{f}$ fermions minimally coupled to the self-dual field and its dual theory will give us a hint on how to answer that question and avoid the ghost problem. 


\section{Matter induced GSD model and its dual}

A seemingly unrelated problem is the coupling of $U(1)$ matter to the self-dual field. In [13] the following master partition function was suggested:

$$
\mathcal{Z}=\int \mathcal{D} \bar{\psi}_{j} \mathcal{D} \psi_{j} \mathcal{D} A \mathcal{D} f \exp ^{i \int d^{3} x \mathcal{L}_{\text {Master }}^{U(1)}}
$$

with

$$
\begin{aligned}
\mathcal{L}_{\text {Master }}^{U(1)} & =\frac{m^{2}}{2} f^{\mu} f_{\mu}+\frac{m}{2} f^{\mu} \epsilon_{\mu \nu \gamma} \partial^{\gamma} f^{\nu}+\bar{\psi}_{j}\left[\not D(f)-m_{f}\right] \psi_{j} \\
& -\frac{m}{2}\left(A^{\mu}-f^{\mu}\right) \epsilon_{\mu \nu \gamma} \partial^{\gamma}\left(A^{\nu}-f^{\nu}\right)
\end{aligned}
$$

The repeated indices are summed over $\left(j=1,2, \cdots, N_{f}\right)$ and $D_{\mu}(f)=\left(i \partial_{\mu}-e f_{\mu} / \sqrt{N_{f}}\right)$. Performing the Gaussian integral over $A^{\mu}$ we have the self-dual model minimally coupled to $N_{f}$ fermionic matter fields:

$$
\mathcal{L}_{S D+M}=\frac{m^{2}}{2} f^{\mu} f_{\mu}+\frac{m}{2} f^{\mu} \epsilon_{\mu \nu \gamma} \partial^{\gamma} f^{\nu}+\bar{\psi}_{j}\left[\not D(f)-m_{f}\right] \psi_{j}
$$

while integrating over $f_{\mu}$ we derive the dual gauge theory which now includes a Pauli-like term and a Thirring term [13] :

$$
\begin{aligned}
\mathcal{L}_{M C S+M}= & -\frac{1}{4} F_{\alpha \beta}(A) F^{\alpha \beta}(A)+\frac{m}{2} \epsilon_{\alpha \beta \gamma} A^{\alpha} \partial^{\beta} A^{\gamma} \\
& -\frac{e}{m} J_{\nu} \epsilon^{\nu \alpha \beta} \partial_{\alpha} A_{\beta}-\frac{e^{2}}{2 m^{2}} J_{\nu} J^{\nu}+\bar{\psi}_{j}\left[i \not \partial-m_{f}\right] \psi_{j} .
\end{aligned}
$$

The equations of motion of (16) are $f_{\mu}=\tilde{f}_{\mu}$ and $\not \partial(f) \psi=0$ while from (17) we obtain $\tilde{A}_{\mu}=\tilde{\tilde{A}}_{\mu}$ and $\not D(\tilde{A}) \psi=0$ where the duality transformation includes now the matter fields $\tilde{A}_{\mu}=-\epsilon_{\mu \nu \gamma} \partial^{\gamma} A^{\nu} / m+\left(e /\left(m^{2} \sqrt{N_{f}}\right)\right) J_{\mu}$ where $J_{\mu}=\bar{\psi}_{j} \gamma_{\mu} \psi_{j}$. Therefore, both theories are dual to each other at classical level through the map $f_{\mu} \leftrightarrow \tilde{A}_{\mu}$, with the matter fields being invariant under the dual map. In [15] we have shown that this dual map can be extended to the quantum level. Once again, those results do not guarantee spectrum equivalence. In particular we have now truly interacting theories which complicates the analysis. Notwithstanding, the integration over the fermions produces a factor $\exp \left[N_{f} \operatorname{tr} \ln \left(i \not \partial-m+e f / \sqrt{N_{f}}\right)\right]$ in the partition function (14). Due to Furry's theorem only even powers of the vector field will survive the $1 / N_{f}$ expansion. Neglecting the field 
independent factor $\exp \left[N_{f} \operatorname{tr} \ln (i \not \partial-m)\right]$ we are left only with the quadratic term of order $e^{2}$ at the leading order in $1 / N_{f}$ which becomes exact at $N_{f} \rightarrow \infty$. The quadratic term is the vacuum polarization diagram. For simplicity we assume four components fermions. By computing the polarization tensor using a gauge invariant regularization, see e.g. [23], we have the effective master action at $N_{f} \rightarrow \infty$ :

$$
\begin{aligned}
\mathcal{L}_{\text {Master }}^{U(1)}\left(N_{f} \rightarrow \infty\right) & =\frac{m^{2}}{2} f^{\mu} f_{\mu}+\frac{m}{2} f^{\mu} \epsilon_{\mu \nu \gamma} \partial^{\gamma} f^{\nu}-\frac{e^{2}}{4} F_{\mu \nu}(f) g(\square) F^{\mu \nu}(f) \\
& -\frac{m}{2}\left(A^{\mu}-f^{\mu}\right) \epsilon_{\mu \nu \gamma} \partial^{\gamma}\left(A^{\nu}-f^{\nu}\right)
\end{aligned}
$$

where, defining $z=-\square / 4 m_{f}^{2}$ we have $g(\square)=f_{2} /\left(16 \pi m_{f}\right)$ with, for $0 \leq z \leq 1$,

$$
f_{2}=\left(\frac{1}{z}-\frac{1+z}{2 z^{3 / 2}} \ln \frac{1+\sqrt{z}}{1-\sqrt{z}}\right)
$$

For $z<0$ the expression for $f_{2}$ can be analytically continued from (19). We neglect the imaginary part of the action which appears above the pair creation threshold $z>1$. Integrating over $A_{\mu}$ we have:

$$
\mathcal{L}_{S D+M}\left(N_{f} \rightarrow \infty\right)=\frac{m^{2}}{2} f^{\mu} f_{\mu}-\frac{m}{2} f^{\mu} \epsilon_{\mu \nu \gamma} \partial^{\gamma} f^{\gamma}-\frac{e^{2}}{4} F_{\mu \nu}(f) g(\square) F^{\mu \nu}(f)
$$

which is of the GSD type with:

$$
a_{0}=\frac{m^{2}}{2} \quad ; \quad a_{1}=-\frac{m}{2} \quad ; \quad a_{2}=-\frac{e^{2} g(\square)}{2}
$$

On the other hand, integrating over $f_{\mu}$ we obtain

$$
\mathcal{L}_{M C A+M}\left(N_{f} \rightarrow \infty\right)=-\frac{m}{2} A^{\mu} \epsilon_{\mu \nu \gamma} \partial^{\gamma} A^{\nu}-\frac{m^{2}}{4} F_{\mu \nu}(A) \frac{1}{\left[m^{2}+e^{2} \square g(\square)\right]} F^{\mu \nu}(A)
$$

which is not of the type (8) with the identifications (21). Therefore, the Noether gauge embedding used at tree level does not go through the effective action at $N_{f} \rightarrow \infty$. Next, we check the particle content of (20). The propagator is of the form (5) with the identification (21). As before we only have to examine the factor:

$$
\frac{\left(a_{0}+k^{2} a_{2}\right)}{\left(a_{0}+k^{2} a_{2}\right)^{2}-a_{1}^{2} k^{2}}=\frac{2 r\left(r+s z f_{2}\right)}{m^{2}\left[\left(r+s z f_{2}\right)^{2}-z\right]}
$$

with the dimensionless couplings $r \equiv m / 2 m_{f} ; s \equiv e^{2} /(4 \pi m)$. Since $r s>0$ and (23) is invariant under $m \rightarrow-m$ it is enough to assume $r>0$ and $s>0$. First of all, we remark 


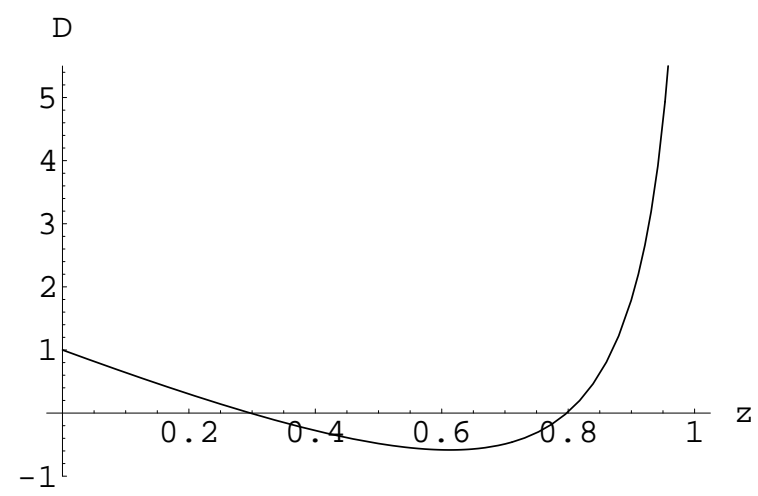

Figure 1: Plot of $D=\left[\left(r+s z f_{2}\right)^{2}-z\right]$ for $r=1=s$.

that for $z<0\left(k^{2}<0\right)$ the denominator of (23) never vanishes since $\lim _{z \rightarrow 0} z f_{2}=0$, which implies that we are always free of tachyons. It is a numerical result that for $0 \leq z \leq 1$ we always have two simple poles. That is $D \equiv\left[\left(r+s z f_{2}\right)^{2}-z\right]=\left(z-z_{+}\right)\left(z-z_{-}\right) R(z)$ where $R(z)$ never vanishes and it is finite except at the pair creation threshold $z \rightarrow 1$ where $z f_{2} \rightarrow \infty$. We have typically the plot given in figure 1 where $z_{+}>z_{-}$.

Noticing that $D=D_{+} D_{-}$with $D_{ \pm}=\left(r+s z f_{2} \pm \sqrt{z}\right)$, we have checked, again numerically, that the poles $z=z_{+}$and $z=z_{-}$come respectively from $D_{+}=0$ and $D_{-}=0$. Consequently, the sign of the residue obtained at those poles can be written respectively in terms of the derivative $\left(D^{\prime}\right)$ as $\pm 2 r \sqrt{z} /\left(m^{2} D^{\prime}\left(z_{ \pm}\right)\right)$. Since, as in figure $1, D^{\prime}\left(z_{+}\right)>0$ and $D^{\prime}\left(z_{-}\right)<0$ both poles are physical and we are free of ghosts for arbitrary values of $e^{2}, m, m_{f}$. In particular, we conclude that the interaction of the self-dual field with the parity invariant fermions has produced, at least at $N_{f} \rightarrow \infty$, an extra massive pole due to the non-local Maxwell term. Now we should verify the spectrum of the dual gauge theory (22). It turns out, after introducing a gauge fixing term, that the propagator obtained from (22) acquires the form:

$$
\left\langle A_{\alpha}(k) A_{\beta}(-k)\right\rangle_{M C S+M}=\frac{g_{\alpha \beta}-\theta_{\alpha \beta}}{\lambda k^{2}}+\frac{\left(a_{0}+k^{2} a_{2}\right)}{\left(a_{0}+k^{2} a_{2}\right)^{2}-a_{1}^{2} k^{2}} \theta_{\alpha \beta}+\frac{i\left(a_{0}+k^{2} a_{2}\right)^{2} E_{\alpha \beta}}{a_{1} k^{2}\left[\left(a_{0}+k^{2} a_{2}\right)^{2}-a_{1}^{2} k^{2}\right]}
$$

where $a_{0}, a_{1}, a_{2}$ are given in (21). Thus, except for the non-physical (gauge dependent) massless pole $k^{2}=0$, which has a vanishing residue [19], as the reader can easily check, choosing a frame $k_{\mu}=(k, k, 0)$ and saturating with conserved currents, $\epsilon^{\mu \nu \alpha} J_{\mu} J_{\nu}^{*} k_{\alpha}=0$. Therefore we have the same particle content of the $\mathcal{L}_{S D+M}$ model at $N_{f} \rightarrow \infty$. In conclusion, the matter induced effective action preserves the particle content without 
artificially creating ghost poles as in the case of the GSD/HD-MCS models in the previous section, although the Lorentz structure of the actions is the same in both cases.

\section{New master action for $a_{2} \neq 0$.}

After the translation $A_{\mu} \rightarrow A_{\mu}+f_{\mu}$ in (13), which has a trivial Jacobian, we have two decoupled theories:

$$
\mathcal{L}_{\text {Master }} \rightarrow \mathcal{L}_{G S D}(f)+a_{1} A^{\mu} \epsilon_{\mu \nu \gamma} \partial^{\gamma} A^{\nu}-\frac{a_{2}}{2} F_{\mu \nu}(A) F^{\mu \nu}(A)
$$

On the other hand, we could decouple the fields through the translation $f_{\mu} \rightarrow f_{\mu}+\tilde{A}_{\mu}$ and obtain:

$$
\mathcal{L}_{\text {Master }} \rightarrow \mathcal{L}_{H D-M C S}(A)+a_{0} f^{\mu} f_{\mu}
$$

Since the spectrum of (25) and (26) must be the same and $f_{\mu}$ in (26) is certainly a nonpropagating field, the poles of $\mathcal{L}_{G S D}(f)$ and of the MCS theory for $A_{\mu}$ in (25) must be contained in $\mathcal{L}_{H D-M C S}(A)$. Indeed, the extra pole we found in (24) is precisely associated with the two last terms in (25) including the non-propagating massless pole. The above translations also explain why a master action gives rise to two theories, in this case $\mathcal{L}_{G S D}(f)$ and $\mathcal{L}_{H D-M C S}(A)$, with different spectrum.

Now it is clear why we did not have a mismatch of spectrum in the matter induced master action. Namely, after a translation $A_{\mu} \Longrightarrow A_{\mu}+f_{\mu}$ in (18) we have:

$$
\mathcal{L}_{\text {Master }}^{U(1)}\left(N_{f} \rightarrow \infty\right) \Longrightarrow \mathcal{L}_{S D+M}\left(f, N_{f} \rightarrow \infty\right)-\frac{m}{2} A^{\mu} \epsilon_{\mu \nu \gamma} \partial^{\gamma} A^{\nu}
$$

If we do a convenient translation in $f_{\mu}$, with trivial Jacobian, we derive instead:

$$
\mathcal{L}_{\text {Master }}^{U(1)}\left(N_{f} \rightarrow \infty\right) \Longrightarrow \mathcal{L}_{M C S+M}\left(A, N_{f} \rightarrow \infty\right)+\frac{m^{2}}{2} f^{\mu} f_{\mu}-\frac{e^{2}}{4} F_{\mu \nu}(f) g(\square) F^{\mu \nu}(f)
$$

Once again the particle content of (27) and (28) must be the same. Since the pure ChernSimons term in (27) contains only one massless non-propagating mode we expect that the poles of $\mathcal{L}_{S D+M}\left(f, N_{f} \rightarrow \infty\right)$ should reappear in (28). We have already checked that the spectrum of $\mathcal{L}_{S D+M}\left(f, N_{f} \rightarrow \infty\right)$ and $\mathcal{L}_{M C S+M}\left(A, N_{f} \rightarrow \infty\right)$ are the same. However, the quadratic terms in the $f_{\mu}$ field in (28) contain apparently a new pole of the type $1 /\left(a_{0}-a_{2} k^{2}\right)$ with $a_{0}, a_{2}$ given in (21). This pole was not present in (27) but it turns out that the propagator of the $\mathcal{L}_{M C S+M}\left(A, N_{f} \rightarrow \infty\right)$ model has exactly a zero, see gauge 
invariant part of (24), at the point $a_{0}-a_{2} k^{2}=0$ such that the poles of (27) and (28) still match as expected. After the above discussions it becomes clear that if we replace the master action given in (13) by the new one below we will have the same spectrum for both dual theories:

$$
\mathcal{L}_{\text {Master }}^{\text {new }}=a_{0} f^{\mu} f_{\mu}+a_{1} \epsilon_{\alpha \beta \gamma} f^{\alpha} \partial^{\beta} f^{\gamma}+\frac{a_{2}}{2} F_{\mu \nu}(f) F^{\mu \nu}(f)-a_{1} \epsilon_{\alpha \beta \gamma}\left(A^{\alpha}-f^{\alpha}\right) \partial^{\beta}\left(A^{\gamma}-f^{\gamma}\right)
$$

Performing a translation $A_{\mu} \rightarrow A_{\mu}+f_{\mu}$ we have a pure Chern-Simons term plus the GSD model given in (11) while a translation in the $f_{\mu}$ field would lead to

$$
\mathcal{L}_{\text {Master }}^{\text {new }} \rightarrow \mathcal{L}_{N L-M C S}+f^{\mu}\left(a_{0} g_{\mu \nu}-a_{2} \square \theta_{\mu \nu}\right) f^{\nu}
$$

where the new action is given by:

$$
\mathcal{L}_{N L-M C S}=-a_{1} \epsilon_{\mu \nu \alpha} A^{\mu} \partial^{\nu} A^{\alpha}-F^{\mu \nu}(A) \frac{a_{1}^{2}}{2\left(a_{0}-a_{2} \square\right)} F_{\mu \nu}(A)
$$

Notice that for $a_{2}=0$ we recover the duality between the SD and MCS models. The corresponding propagator is the same as (24). Thus, we do not need to check that the spectrum of $\mathcal{L}_{N L-H D-M C S}$ and $\mathcal{L}_{G S D}$ is the same, that is, two physical massive particles without ghosts. Now let us check the dual map. The new master action furnishes the equations of motion:

$$
\begin{aligned}
d(A-f) & =0 \\
f_{\mu} & =\tilde{f}_{\mu}
\end{aligned}
$$

The duality transformation is defined as in section 2. The equation (33) is the same of the GSD model. From (32) and (33) we have $A_{\mu}+\partial_{\mu} \phi=\tilde{A}_{\mu}$ which leads to $a_{2}^{2}\left(\square+m_{+}^{2}\right)\left(\square+m_{-}^{2}\right) \tilde{A}_{\mu}=0$. Since the dual of a total divergence vanishes we have $\tilde{A}_{\mu}=\tilde{\tilde{A}}_{\mu}$ which guarantees the classical duality between $\mathcal{L}_{N L-M C S}$ and $\mathcal{L}_{G S D}$ using the map $f_{\mu} \leftrightarrow \tilde{A}_{\mu}$. On the other hand, from (13) we have:

$$
\begin{aligned}
\tilde{f}_{\mu} & =\tilde{A}_{\mu} \\
f_{\mu} & =\tilde{f}_{\mu}
\end{aligned}
$$

They also lead to $\tilde{A}_{\mu}=\tilde{\tilde{A}}_{\mu}$. If $a_{2}=0, \mathcal{L}_{\text {Master }}^{\text {new }}$ and $\mathcal{L}_{\text {Master }}$ are equivalent as (32) and (34). In the following we confirm that the dual equivalence persists at quantum level up to contact terms. If we define the generating function: 


$$
\mathcal{Z}(J)=\int \mathcal{D} A \mathcal{D} f e^{i \int d^{3} x\left[\mathcal{L}_{\text {Master }}^{\text {New }}(f, A)+\frac{\lambda\left(\partial_{\mu} A^{\mu}\right)^{2}}{2}+J^{\alpha} \tilde{A}_{\alpha}\right]}
$$

After $A_{\mu} \rightarrow A_{\mu}+f_{\mu}$ and integrating over $A^{\mu}$ we obtain, up to an overall constant,

$$
\mathcal{Z}(J)=\int \mathcal{D} f e^{i \int d^{3} x\left[\mathcal{L}_{G S D}(f)+J^{\alpha} D_{\alpha \beta} J^{\beta}+J^{\alpha} \tilde{f}_{\alpha}\right]}
$$

with

$$
D_{\alpha \beta}=\frac{-1}{4 a_{1} a_{0}^{2}}\left[\left[\left(a_{2}^{2} \square-a_{1}^{2}\right) E_{\alpha \beta}-2 a_{1} a_{2} \square \theta_{\alpha \beta}\right]\right.
$$

On the other side, if we integrate over $f_{\mu}$ in (36) we have $\mathcal{L}_{N L-H D-M C S}+\frac{\lambda\left(\partial_{\mu} A^{\mu}\right)^{2}}{2}+J^{\alpha} \tilde{A}_{\alpha}$. Therefore, deriving with respect to $J_{\alpha}$ we deduce:

$$
\left\langle\tilde{f}_{\alpha}(x) \tilde{f}_{\beta}(y)\right\rangle_{G S D}=\left\langle\tilde{A}_{\alpha}(x) \tilde{A}_{\beta}(y)\right\rangle_{N L-M C S}-D_{\alpha \beta} \delta^{(3)}(x-y)
$$

Furthermore, if we define the generating function

$$
\mathcal{Z}_{G S D}(J, j)=\int \mathcal{D} f e^{i \int d^{3} x\left(\mathcal{L}_{G S D}(f)+J^{\alpha} \tilde{f}_{\alpha}+j^{\alpha} f_{\alpha}\right)}
$$

and make $f_{\mu} \rightarrow f_{\mu}+J_{\mu} / 2 a_{0}$ we have

$\mathcal{Z}_{G S D}(J, j)=\int \mathcal{D} f \exp \left\{i \int d^{3} x\left[\mathcal{L}_{G S D}(f)+\left(j^{\alpha}+J^{\alpha}\right) f_{\alpha}-\frac{J_{\nu} j^{\nu}}{2 a_{0}}-\frac{J_{\mu} H^{\mu \beta} J_{\beta}+J_{\nu} J^{\nu}}{4 a_{0}}\right]\right\}$

Where $H_{\alpha \beta}$ is the operator on the right handed side of (2), i.e., $\tilde{f}_{\alpha} \equiv H_{\alpha \beta} f^{\beta}$. From (40) and (41), see also [24, 18], we have

$$
\begin{aligned}
\left\langle f_{\alpha}(x) \tilde{f}_{\beta}(y)\right\rangle_{G S D} & =\left\langle f_{\alpha}(x) f_{\beta}(y)\right\rangle_{G S D}+\frac{g_{\alpha \beta}}{2 a_{0}} \delta^{(3)}(x-y) \\
\left\langle\tilde{f}_{\alpha}(x) \tilde{f}_{\beta}(y)\right\rangle_{G S D} & =\left\langle f_{\alpha}(x) f_{\beta}(y)\right\rangle_{G S D}+\frac{\left(g_{\alpha \beta}+H_{\alpha \beta}\right)}{2 a_{0}} \delta^{(3)}(x-y)
\end{aligned}
$$

Since the GSD model is quadratic the relations (42) and (43) assure that the classical self-duality $f_{\mu}=\tilde{f}_{\mu}$ holds also at quantum level up to contact terms. Combining (39) and (43) we derive:

$$
\left\langle f_{\alpha}(x) f_{\beta}(y)\right\rangle_{G S D}=\left\langle\tilde{A}_{\alpha}(x) \tilde{A}_{\beta}(y)\right\rangle_{N L-M C S}-\left(D_{\alpha \beta}-\frac{\left(g_{\alpha \beta}+H_{\alpha \beta}\right.}{2 a_{0}}\right) \delta^{(3)}(x-y)
$$


We conclude that $\mathcal{L}_{N L-M C S}(A)$ is dual to $\mathcal{L}_{G S D}(f)$ at classical and quantum level (up to contact terms) with the map $f_{\mu} \leftrightarrow \tilde{A}_{\mu}$. From this point of view $\mathcal{L}_{N L-M C S}(A)$ is on the same footing of $\mathcal{L}_{H D-M C S}(A)$ but it has the advantage of having the same spectrum of the GSD model and being ghost free.

We end up this section with three remarks. First, it is tempting to formally redefine the gauge field $A_{\mu} \rightarrow\left(a_{0}-a_{2} \square\right)^{1 / 2} A_{\mu}$ in order to make $\mathcal{L}_{N L-M C S}(A)$ a local gauge theory. However, the higher derivative theory thus obtained, although classically equivalent to the GSD model and simpler than $\mathcal{L}_{H D-M C S}$, contains two poles $k^{2}=m_{ \pm}^{2}$ and one of them is necessarily a ghost now. This is not totally surprising, the situation is similar to the massless Schwinger model in $1+1$ dimensions where the integration over the fermionic massless fields gives rise to the effective (exact) gauge invariant action:

$$
\mathcal{L}=-\frac{1}{4} F_{\mu \nu}^{2}-\frac{e^{2}}{4 \pi} F^{\mu \nu} \frac{1}{\square} F_{\mu \nu}
$$

The photon propagator from the above Lagrangian contains only one massive pole at $k^{2}=e^{2} / \pi$ and no ghosts. However, if we try a naive redefinition $A_{\mu} \rightarrow \sqrt{ \pm \square} A_{\mu}$ the action becomes local but the propagator will get a factor $\pm\left[k^{2}\left(k^{2}-e^{2} / \pi\right)\right]^{-1}$. Whatever sign we choose we always have a ghost field either at $k^{2}=0$ or $k^{2}=e^{2} / \pi$. In fact in this specific case we can achieve a consistent local formulation by introducing a scalar field through $A_{\mu}=\left(\epsilon_{\mu \nu} \partial^{\nu} / \sqrt{\square}\right) \phi$. However, a consistent formulation in terms of a vector gauge field can only be non-local to the best we know.

The second remark concerns the uniqueness of the new master action. Clearly, the important point in our proposal is that the gauge and the non-gauge fields are mixed though a non-propagating term, in this case of $d=2+1$ we have used the Chern-Simons term. One might try to generalize the new master action by using an arbitrary constant in front of the mixed Chern-Simons term in (29) instead of $a_{1}$. We have done that and checked that all important conclusions about dual equivalence, spectrum match, absence of ghosts, etc, hold for arbitrary values of this parameter but the simplest dual gauge theory is obtained precisely for the theory suggested here.

At last, we remark that the duality between $\mathcal{L}_{N L-M C S}(A)$ and the GSD model obtained by the new master action is also valid for the case where $a_{0}=a_{0}(\square), a_{2}=a_{2}(\square)$ are not constants. If the coefficient of the Chern-Simons mixing term $a_{1}$ is kept constant we expect once again a spectrum equivalence otherwise the match of the spectrum between the non-gauge and the gauge theory will no longer be true in general. 


\section{Conclusion}

Recently, the Noether gauge embedding and the master action procedures were applied in different theories and different dimensions. In particular, very recently, one has been trying to generalize them to nonabelian theories [25] and models defined on the noncommutative plane [26]. This embedding procedure usually reproduces the result of a master action which is very convenient at quantum level. Here we have shown how we can modify this master action to avoid extra poles in the propagator and assure that both dual theories have the same spectrum. Though, we have used a generalized self-dual model as an example, it is clear that similar ideas can be useful whenever we make use of interpolating master actions. The key point is to choose a non-propagating term to mix the gauge and non-gauge fields. To lend support to our suggestion we have compared the spectrum of the effective action generated by the minimal coupling of fermions (parity invariant) to the self-dual field and the effective action generated by its dual gauge theory, both in the limit $N_{f} \rightarrow \infty$ where the quadratic effective actions become exact. As a by product we have shown that those theories contain two massive poles in their spectrum and are free of ghosts and tachyons ${ }^{2}$. The price we have paid for a ghost free dual gauge theory was locality. However, it is well know that it is not easy to have massive vector fields and still keep local gauge invariance, sometimes we have to break locality as in the massless Schwinger model. Since the GSD model contains two massive vector modes, one might speculate that the usual topological mechanism [27] to generate mass for a gauge field in $d=2+1$, with the help of higher derivatives, could not cope with two massive poles without generating unwanted extra poles. More work is necessary to clarify this point.

\section{Acknowledgements}

This work was partially supported by $\mathbf{C N P q}$, a Brazilian research agency. We thank Alvaro de Souza Dutra for discussions and bringing reference 22 to our attention. A discussion with Marcelo Hott is also acknowledged.

\section{References}

[1] S. Coleman, Phys. Rev. D11 (1975) 2088, Ann. Phys. 101 (1976) 239.

\footnotetext{
${ }^{2}$ Clearly, a more general matter coupling, including even a non-minimal coupling with scalar fields also deserves to be examined but that is beyond the aims of this letter.
} 
[2] S. Mandelstam, Phys. Rev. D 11 (1975) 3026.

[3] E. Abdalla, M. C. Abdalla and K. D. Rothe, "Non-perturbative methods in two dimensional quantum field theory". World Scientific 1991 - Singapore.

[4] N. Seiberg and E. Witten, Nucl. Phys. B 246 (1994) 19.

[5] S. Deser, R. Jackiw, Phys. Lett. B139 (1984) 371.

[6] P.K. Townsend, K. Pilch and P. van Nieuwenhuizen, Phys. Lett B 136 (1984)38.

[7] E. Fradkin and F. A. Schaposnik, Phys. Lett. B 338 (1994) 253. G. Rossini and F. A. Schaposnik, Phys. Lett. B 338 (1994) 465.

[8] Kei-Ichi Kondo, Progr. Theor. Phys. 94 (1995) 899.

[9] R. Banerjee and E. C. Marino, Mod. Phys. Lett. A14 (1999) 593.

[10] D.G. Barci, L.E. Oxman and S.P. Sorella, Phys. Rev. D 59 (1999) 105012.

[11] D. Dalmazi, A. de Souza Dutra and M. Hott, Phys. Rev. D 67 (2003) 125012.

[12] M. Botta Cantcheff and J. A. Helayel-Neto, Phys. Rev. D 67 (2003) 025016.

[13] M. Gomes, L. C. Malacarne and A. J. da Silva, Phys. Lett. B439 (1998) 137.

[14] M.A. Anacleto, A. Ilha, J.R.S. Nascimento, R.F. Ribeiro and C. Wotzasek, Phys. Lett. B504 (2001) 268.

[15] D. Dalmazi, Journal of Phys. A37 (2004) 2487.

[16] R. Menezes, J.R.S. Nascimento, R.F. Ribeiro and C. Wotzasek, Phys. Lett. B537 (2002) 321.

[17] D. Bazeia, A. Ilha, J.R.S. Nascimento, R.F. Ribeiro and C. Wotzasek, Phys. Lett. B510 (2001) 329.

[18] D. Bazeia, R. Menezes, J.R. Nascimento, R.F. Ribeiro and C. Wotzasek, Journal of Phys. A36 (2003) 9943.

[19] A.P. Baêta Scarpelli, M. Botta Cantcheff and J.A. Helayel-Neto, Europhysics Lett. 65 (2003) 760. 
[20] M. Botta Cantcheff, C.F. Godinho, A.P. Baeta Scarpelli and J.A. Helayel-Neto, Phys.Rev. D68 (2003) 65025.

[21] O.M. Del Cima, Int. Journal of Mod. Phys. A10 (1995) 1641.

[22] A. de Souza Dutra and C.P. Natividade, Mod.Phys.Lett A11 (1996) 775-783.

[23] D. Dalmazi, Phys. Rev. D70 (2004) 065021.

[24] R. Banerjee, H.J. Rothe and K. D. Rothe, Phys. Rev. D 52 (1995) 3750.

[25] A. Ilha and C. Wotzasek, Nucl. Phys. B604 (2001) 426, M. Botta Cantcheff, Phys.Lett. B528 (2002) 283-287, D. C. Rodrigues and C. Wotzasek, hep-th/0305141, T.Mariz, R. Menezes, J.R.S. Nascimento, R.F.Ribeiro, C. Wotzasek, het-th/0306265.

[26] S.Gosh, Phys. Lett. B558 (2003) 245 ; M.B. Cantcheff anf P. Minces, Eur. Phys. J. C34 (2004) 393; T.Mariz, R. Menezes, J.R.S. Nascimento, R.F. Ribeiro and C. Wotzasek, Phys. Rev. D70 (2004) 085018; E. Harikumar and Victor O. Rivelles, hept-th/0506078

[27] S. Deser, R. Jackiw and S. Templeton, Ann. of Phys. 140(1982) 372. 